05,11

\title{
Использование усреднения по полям взаимодействия для построения приближенных методов в модели Изинга разбавленного магнетика
}

\author{
(C) С.В. Сёмкин, В.П. Смагин, В.И. Люлько \\ Владивостокский государственный университет экономики и сервиса, \\ Владивосток, Россия \\ E-mail: Li15@rambler.ru
}

Поступила в Редакцию 5 февраля 2020 г.

В окончательной редакции 5 февраля 2020 г.

Принята к публикации 17 марта 2020 г.

\begin{abstract}
Проанализировано применение метода усреднения по полям взаимодействия применительно к задачам статистической физики. Построено теоретическое обоснование метода усреднения по полям взаимодействия применительно к кластеру спинов. На основе полученных общих соотношений найдены приближенные решения для модели Изинга с разбавлением по связям. Эти приближенные решения сравниваются с точным решением для одномерной цепочки изинговских спинов с разбавлением по связям.
\end{abstract}

Ключевые слова: модель Изинга, поле взаимодействия, разбавленный магнетик, решетка Бете.

DOI: $10.21883 /$ FTT.2020.08.49603.021

\section{1. Введение}

Известно, что свойства разбавленных и неупорядоченных магнетиков отличаются от свойств чистых магнетиков $[1-3,10]$. Однако точных решений для моделей магнитных систем с разбавлением до сих пор не получено. Поэтому имеет смысл построение приближенных решений для разбавленных магнетиков. Некоторые из этих решений можно построить с помощью усреднения по полям взаимодействия.

Применение функции распределения по полям взаимодействия к изучению свойств системы многих взаимодействующих частиц началось с работы Чандрасекара [4]. В этой работе рассматривалось движение системы галактик, связанных гравитационным взаимодействием. Позднее этот метод был использован для оценки влияния магнитостатического взаимодействия мелких ферримагнитных частиц, находящихся в немагнитной матрице [5]. В работах [6-8] метод усреднения по обменным полям был применен к анализу магнитных свойств чистых и разбавленных магнетиков. Применение метода к задачам статистической физики было основано на работе [9], в которой показано, что для модели Изинга в состоянии термодинамического равновесия для любого спина $\sigma_{0}$

$$
\left\langle\sigma_{0}\right\rangle=\left\langle\operatorname{th}\left(K h+h_{e x}\right)\right\rangle,
$$

где $K=J / k T$ и $h_{e x}=H_{e x} / k T, J$ - константа обменного взаимодействия, $H_{e x}-$ внешнее магнитное поле, $k-$ постоянная Больцмана, $T$ - температура. Поле взаимодействия $h$ является в данном случае суммой всех спинов, взаимодействующих с $\sigma_{0}$.

В настоящей работе мы сформулируем и докажем соотношения (формула (2)), на которых может быть основан метод усреднения по обменным полям применительно к кластерам из нескольких спинов. Применив полученные соотношения к модели Изинга с разбавлением по связям, мы построим для этой модели (в качестве примера) два варианта приближенных методов. Эти методы мы проверяем на одномерной модели Изинга с разбавлением по связям, для которой мы построили точное решение [17]. Кроме того, мы сравниваем полученную в этих приближениях намагниченность при нулевой температуре с вероятностью того, что узел разбавленной по связям решетки Бете принадлежит бесконечному кластеру.

\section{2. Усреднение по полям взаимодействия}

Рассмотрим систему из $N$ взаимодействующих частиц, каждая из которых характеризуется некоторым параметром $\sigma$, который в дальнейшем, имея в виду применение к модели Изинга, будем называть спином. Обозначим $\Omega$ множество всех этих спинов, а гамильтониан системы - $\mathscr{H}(\Omega)$. Конкретный вид гамильтониана значения не имеет, но мы будем полагать, что для каждого спина $\sigma_{i}$ в гамильтониане есть конечное число слагаемых, содержащих $\sigma_{i}$. Причем это число остается конечным и в термодинамическом пределе $N \rightarrow \infty$. Два спина $\sigma_{i}$ и $\sigma_{j}$ будем называть взаимодействующими, если в гамильтониане есть слагаемое не аддитивно зависящее от $\sigma_{i}$ и $\sigma_{j}$.

Рассмотрим в системе группу, содержащую $n$ спинов. Такую группу мы будем в дальнейшем называть кластером. Множество входящих в кластер спинов будем обозначать $c$. Обозначим $r$ множество не входящих в 
кластер спинов, каждый из которых взаимодействует хотя бы с одним спином кластера, и обозначим $s$ множество всех остальных спинов. Очевидно, $\Omega$ является объединением непересекающихся множеств $c, r$ и $s$.

Выделим теперь в гамильтониане слагаемые, связанные с взаимодействием спинов принадлежащих $c$ и $r$

$$
\mathscr{H}(\Omega)=\mathscr{H}_{c}(c, r)+\mathscr{H}_{s}(r, s)
$$

Гамильтониан $\mathscr{H}_{c}(c, r)$ содержит только слагаемые, зависящие от спинов кластера $c$ и слагаемые, описывающие взаимодействие спинов кластера со спинами из множества $r$. Гамильтониан $\mathscr{H}_{s}(r, s)$ - содержит все остальные слагаемые, входящие в $\mathscr{H}(\Omega)$. Тогда статистическую сумму системы можно записать в виде

$$
Z=\sum_{r} Z_{c}(r) Z_{S}(r)
$$

где

$$
Z_{s}(r)=\sum_{s} \exp \left(-\frac{1}{k T} \mathscr{H}_{s}(r, s)\right)
$$

и

$$
Z_{c}(r)=\sum_{c} \exp \left(-\frac{1}{k T} \mathscr{H}_{c}(c, r)\right) .
$$

Каждое слагаемое в (1) имеет смысл (ненормированной) вероятности того, что система спинов $r$ будет находиться в некотором состоянии. Нормированная вероятность $W(r)=Z_{c}(r) Z_{s}(r) / Z$ есть функция распределения для наборов состояний спинов, взаимодействующих с кластером.

Пусть $f(r)$ некоторая функция спинов, принадлежащих $r$, а $\varphi(c)$ некоторая функция кластерных спинов $c$. Тогда среднее по ансамблю значение произведения $f \varphi$ можно представить в виде

$$
\langle f \varphi\rangle=\frac{1}{Z} \sum_{r} f(r)\left(\sum_{c} \varphi(c) \exp \left(-\frac{1}{k T} \mathscr{H}_{c}(c, r)\right)\right) Z_{s}(r) .
$$

Разделив и умножив каждое слагаемое в этой сумме на „кластерную“ статсумму $Z_{c}(r)$, запишем полученное выражение в виде

$$
\langle f \varphi\rangle=\sum_{r} f(r)\langle\varphi\rangle_{r} W(r)
$$

где

$$
\langle\varphi\rangle_{r}=\frac{1}{Z_{c}(r)} \sum_{c} \varphi(c) \exp \left(-\frac{1}{k T} \mathscr{H}_{c}(c, r)\right) .
$$

Формулу (2) можно интерпретировать следующим образом. Выражение (3) можно понимать как „кластерное среднее“ функции $\varphi(c)$, вычисленное при условии, что конфигурация взаимодействующих с кластером спинов задана и неизменна. Выражение (2) в этом случае можно понимать как усреднение произведения $f(r)\langle\varphi\rangle_{r}$ по функции распределения $W(r)$. На использовании формулы (2) и основан метод усреднения по полям взаимодействия.

Рассмотрим модель Изинга на некоторой решетке. Этой решеткой может быть какая-нибудь периодическая решетка с координационным числом $q$, но, в принципе, под словом „решетка“ можно понимать произвольный граф (не содержащий, разумеется, петель и параллельных ребер). Пусть в каждом узле решетки содержится изинговский „спин“ принимающий значения +1 и -1 , а взаимодействуют только спины, находящиеся в связанных узлах. Тогда гамильтониан модели Изинга можно записать так:

$$
\mathscr{H}(\Omega)=-J \sum_{(i, j)} \sigma_{i} \sigma_{j}-H_{e x} \sum_{i} \sigma_{i}
$$

Суммирование в первой сумме проводится по всем парам связанных спинов, во второй - по всем узлам.

Выделим теперь на решетке кластер, состоящий только из одного спина $\sigma_{0}$. Кластерный гамильтониан $\mathscr{H}_{c}(c, r)=-J \sigma_{0} \sum_{\sigma_{i} \in r} \sigma_{i}-\sigma_{0} \mathscr{H}_{e x}$, а формула (2), в которой примем $f=1$ и $\varphi=\sigma_{0}$, приводит к

$$
\left\langle\sigma_{0}\right\rangle=\sum_{r} \operatorname{th}\left(K \sum_{\sigma_{i} \in r} \sigma_{i}+h_{e x}\right) W(r),
$$

что совпадает с полученным в [9].

Для простой решетки средние значения каждого спина равны между собой и равны $M$-макроскопической намагниченности системы. В более сложных случаях средние значения (5) (и функции распределения $W(r)$ ) могут быть различны для разных спинов.

Условная вероятность (5) является функцией суммы значений спинов, принадлежащих $r$, то есть суммы спинов, непосредственно взаимодействующих с $\sigma_{0}$. Будем называть эту сумму „полем взаимодействия“ и обозначим $h$, усреднение в (5) является, в сущности, усреднением по функции распределения этого поля взаимодействия

$$
\left\langle\sigma_{0}\right\rangle=\int \operatorname{th}\left(K h+h_{e x}\right) W(h) d h,
$$

где

$$
W(h)=\sum_{r} \delta\left(h-\sum_{\sigma_{i} \in r} \sigma_{i}\right) W(r),
$$

здесь $\delta$ - дельта-функция.

Таким образом, среднюю намагниченность каждого конкретного спина в модели Изинга на любой решетке можно рассматривать как среднее значение th $\left(K h+h_{e x}\right)$ по функции распределения „полей взаимодействия“ $W(h)$, вычисленной для этого спина. 
Возьмем теперь кластер из двух соседних спинов $\sigma_{1}$ и $\sigma_{2}$ (димер). Взяв в (2) $f=1$ и $\varphi=\frac{\sigma_{1}+\sigma_{2}}{2}$, получим

$$
\begin{aligned}
& \left\langle\frac{\sigma_{1}+\sigma_{2}}{2}\right\rangle \\
& =\int \frac{\operatorname{sh}\left(K\left(h_{1}+h_{2}\right)+2 h_{e x}\right)}{\operatorname{ch}\left(K\left(h_{1}+h_{2}\right)+2 h_{e x}\right)+e^{-2 K} \operatorname{ch}\left(K\left(h_{1}-h_{2}\right)\right)} \\
& \quad \times W\left(h_{1}, h_{2}\right) d h_{1} d h_{2},
\end{aligned}
$$

где $h_{1,2}-$ поля взаимодействия, то есть суммы спинов из множества $r$, взаимодействующих со спинами $\sigma_{1}$ и $\sigma_{2}$ соответственно. Функция распределения этих полей $W\left(h_{1}, h_{2}\right)$ определяется аналогично (6). Для простой решетки средние (8) одинаковы для каждого димера и равны макроскопической намагниченности системы на атом.

Рассмотрим теперь модель Изинга на некоторой простой решетке, но с немагнитным разбавлением по связям. (Приводимые ниже рассуждения можно, с небольшими изменениями, распространить и на случай разбавления по узлам.) Будем полагать, что связь исходной решетки с вероятностью $1-b$ разорвана, то есть слагаемое, описывающее взаимодействие спинов по этой связи исключено из гамильтониана (4). Будем считать, что разорванные связи случайно и без корреляции разбросаны по узлам решетки. Рассматривая каждый отдельный $i$-й магнитный атом в такой решетке и применив для него равенство (6), получим, что среднее значение его спина равно

$$
\left\langle\sigma_{i}\right\rangle=\int \operatorname{th}\left(K h+h_{e x}\right) W(h) d h
$$

где $W(h)$,локальная“ функция распределения по полям взаимодействия на этом конкретном атоме. Согласно методу самоусреднения [10], для вычисления макроскопической намагниченности $M$ разбавленного магнетика нужно вычислить среднее всех $\left\langle\sigma_{i}\right\rangle$ в системе с достаточно большим $N$ (точнее, в термодинамическом пределе):

$$
M=\overline{\left\langle\sigma_{i}\right\rangle}=\int \operatorname{th}\left(K h+h_{e x}\right) W(h, b) d h,
$$

где $W(h, b)=\overline{W(h)}$ - „средняя“ функция распределения полей взаимодействия. Формулу (9) можно обобщить и на произвольный кластер, однако следует иметь в виду, что связи внутри самого кластера могут оказаться разорванными. Поэтому „самоусреднение“ необходимо проводить отдельно для каждой конфигурации разорванных связей в кластере, а затем усреднить по этим конфигурациям с весами, равными вероятности соответствующих конфигураций. Например, для кластера из двух узлов есть две конфигурации - с разорванной и не разорванной связью между атомами кластера. Вероятности этих конфигураций есть $1-b$ и $b$ соответственно.
Значит

$$
\begin{aligned}
& M=\overline{\left\langle\frac{\sigma_{1}+\sigma_{2}}{2}\right\rangle}=(1-b) \int \frac{1}{2}\left(\operatorname{th}\left(K h_{1}+h_{e x}\right)\right. \\
& \left.+\operatorname{th}\left(K h_{2}+h_{e x}\right)\right) W_{1}\left(h_{1}, h_{2}, b\right) d h_{1} d h_{2} \\
& +b \int \frac{\operatorname{sh}\left(K\left(h_{1}+h_{2}\right)+2 h_{e x}\right)}{\operatorname{ch}\left(K\left(h_{1}+h_{2}\right)+2 h_{e x}\right)+e^{-2 K} \operatorname{ch}\left(K\left(h_{1}-h_{2}\right)\right)} \\
& \times W_{2}\left(h_{1}, h_{2}\right) d h_{1} d h_{2},
\end{aligned}
$$

Средние функции распределения $W_{1}\left(h_{1}, h_{2}, b\right)$ и $W_{2}\left(h_{1}, h_{2}, b\right)$ получаются усреднением по кластерам с разорванной и не разорванной связью соответственно.

\section{3. Приближенные методы}

Вычисление намагниченности в модели Изинга с разбавлением непосредственно по формулам (9) или (10) возможно, конечно же, только если известны соответствующие функции распределения полей взаимодействия и как видно из этих формул, нахождение функций распределения фактически эквивалентно точному решению задачи. Однако формулы (9) и (10) (или формулы, которые можно аналогичным способом получить из (2) для кластеров с большим числом атомов) можно использовать для построения приближенных методов нахождения намагниченности в модели Изинга с разбавлением. Для этого нужно принять те или иные приближения для функций распределения $W, W_{1}$ и $W_{2}$, входящих в формулы (9) и (10). В настоящей работе мы рассмотрим два варианта приближений и оценим их точность.

Самым простым (и грубым) приближением представляется замена усреднения по полям в (9) и (10) подстановкой средних значений этих полей. Иными словами, функции распределения в (9) и (10) возьмем в следующем виде:

$$
W(h, b)=\delta\left(h-h_{a}\right)
$$

И

$$
W_{1}\left(h_{1}, h_{2}, b\right)=W_{2}\left(h_{1}, h_{2}, b\right)=\delta\left(h_{1}-h_{1 a}\right) \delta\left(h_{2}-h_{2 a}\right) .
$$

В отношении средних полей $h_{a}, h_{1 a}$ и $h_{2 a}$ примем следующие предположения. Будем считать $h_{1 a}=h_{2 a}$, а величины полей будем считать пропорциональными количеству внешних соседних узлов: $h_{1 a} / h_{a}=(q-1) / q$. Обозначив $\mathrm{x}$ коэффициент этой пропорциональности, из (9) и (10) получим

$$
M=\operatorname{th}\left(K q x+h_{e x}\right),
$$

где $x$ определяется из уравнения

$$
\begin{aligned}
\operatorname{th}(K q x & \left.+h_{e x}\right)=(1-b) \operatorname{th}\left(K(q-1) x+h_{e x}\right) \\
& +b \frac{\operatorname{sh}\left(2 K(q-1) x+2 h_{e x}\right)}{\operatorname{ch}\left(2 K(q-1) x+2 h_{e x}\right)+e^{-2 K}}
\end{aligned}
$$


Оказывается [11], что приближение (11) для чистого магнетика $(b=1)$ является точным решением для модели Изинга на решетке Бете, а при $b<1$ его можно рассматривать как „псевдохаотическое“ приближение для модели Изинга с немагнитным разбавлением на решетке Бете [12]. (Псевдохаотическое приближение получается из решения задачи с подвижными немагнитными примесями при наложении дополнительного условия равенства нулю корреляции в расположении примесей в соседних узлах решетки [13].)

Другое приближение для функций распределения, входящих в (8) и (9), состоит в следующем [18]. У каждого $i$-го узла разбавленной по связям решетки есть $k$ связанных с этим узлом соседей. Это случайное число, принимающее значения от 0 до $q$ и при случайном и некоррелированном расположении разорванных связей в решетке распределенное по биноминальному закону. Следовательно, функцию $W(h, b)$, входящую в (9), всегда можно представить в виде

$$
W(h, b)=\sum_{k=0}^{q} C_{q}^{k} b^{k}(1-b)^{q-k} W^{(k)}(h, b) .
$$

Здесь $W^{(k)}(h, b)$ - условная функция распределения по полям взаимодействия при условии, что у узла есть ровно $k$ связанных с ним соседних узлов, $C_{q}^{k}-$ биноминальные коэффициенты. Аналогично, функции $W_{1,2}\left(h_{1}, h_{2}, b\right)$, входящие в выражение (10), можно представить в виде

$$
\begin{aligned}
W_{1,2}\left(h_{1}, h_{2}, b\right)= & \sum_{p=0}^{q-1} \sum_{l=0}^{q-1} C_{q-1}^{p} C_{q-1}^{l} b^{p+l}(1-b)^{2(q-1)-(p+l)} \\
& \times W_{1,2}^{(p, l)}\left(h_{1}, h_{2}, b\right) .
\end{aligned}
$$

Построим теперь следующее приближение. Заменим усреднение по условным функциям распределения $W^{(k)}$ и $W_{1,2}^{(p, l)}$ подстановкой в соответствующие выражения условных средних. Иными словами возьмем условные функции распределения в виде

$$
W^{(k)}(h, b)=\delta\left(h-h_{a}^{(k)}\right)
$$

и

$W_{1}^{(p, l)}\left(h_{1}, h_{2}, b\right)=W_{2}^{(p, l)}\left(h_{1}, h_{2}, b\right)=\delta\left(h_{1}-h_{1 a}^{(p)}\right) \delta\left(h_{2}-h_{2 a}^{(l)}\right)$.

Будем считать, что средние значения полей $h_{a}^{(k)}, h_{1 a}^{(p)}$ и $h_{2 a}^{(l)}$ пропорциональны значениям $k, p$ и $l$ соответственно с одним и тем же коэффициентом пропорциональности, который обозначим $y$. Тогда из (9) и (10) получим

$$
M=\sum_{k=0}^{q} C_{q}^{k} b^{k}(1-b)^{q-k} \operatorname{th}\left(K k y+h_{e x}\right),
$$

где $y$ определяется из уравнения

$$
\begin{aligned}
& \sum_{k=0}^{q} C_{q}^{k} b^{k}(1-b)^{q-k} \operatorname{th}\left(K k y+h_{e x}\right) \\
= & (1-b) \sum_{p=0}^{q-1} C_{q-1}^{p} b^{p}(1-b)^{q-1-p} \operatorname{th}\left(K p y+h_{e x}\right) \\
& +b \sum_{p=0}^{q-1} \sum_{l=0}^{q-1} C_{q-1}^{p} C_{q-1}^{l} b^{p+l}(1-b)^{2(q-1)-(p+l)} \\
& \times \frac{\operatorname{sh}\left(K(p+l) y+2 h_{e x}\right)}{\operatorname{ch}\left(K(p+l) y+2 h_{e x}\right)+e^{-2 K} \operatorname{ch}(K(p-l) y)} .
\end{aligned}
$$

Приближение (13) в дальнейшем будем называть „биноминальным“. Рассмотрим основные свойства биноминального приближения (13). Во-первых, заметим, что для чистого магнетика $(b=1)$ приближение (13) совпадает с (11) и является, таким образом, точным решением для модели Изинга на решетке Бете. Есть еще одно сходство между приближениями (13) и (11). А именно, если для приближения (11) найти критическое значение параметра $K=K_{c}$, при котором исчезает ненулевой корень $(12)$ для $h_{e x}=0$, получим $[11,12]$

$$
K_{c}=\frac{1}{2} \ln \frac{b+b_{c}}{b-b_{c}}, \quad b_{c}=\frac{1}{q-1} .
$$

Используя (14), нетрудно показать, что в биноминальном приближении для критического значения параметра $K_{c}$ и перколяционного порога $b_{c}$ получается точно такой же результат (15). Однако, в общем случае приближения (11) и (13) не совпадают. Для того, чтобы сопоставить эти приближения по степени точности, их, конечно, желательно сравнить с точным решением. Для модели Изинга с разбавлением по узлам или связям точное решение можно построить для одномерной цепочки [14]. Как известно [15], одномерная цепочка является в некотором смысле „патологическим“ видом кристаллической решетки - в ней не может быть фазового перехода и спонтанной намагниченности при ненулевой температуре, а при немагнитном разбавлении бесконечный кластер магнитных атомов разрушается при любой ненулевой концентрации примесей. То есть, рассматривая одномерную цепочку с немагнитными примесями, мы всегда находимся в области концентраций ниже порога протекания и температур выше температуры Кюри. Тем не менее, возможность получить точное решение позволяет сопоставить его с приближенными методами.

Рассмотрим одномерный изинговский магнетик (цепочку) с разбавлением по связям. При таком разбавлении цепочка разбивается на отрезки магнитных атомов разной длины, разделенные разорванными связями. Среднее значение изинговского спина, в расчете на один 
магнитный атом, может быть вычислено так:

$$
M=\sum_{n=1}^{\infty} m_{n} p_{n}
$$

где $m_{n}-$ средняя намагниченность атома отрезка длиной $n$, а $p_{n}-$ вероятность того, что произвольно взятый магнитный атом принадлежит такому отрезку. Очевидно, что $p_{n}=n b^{n-1}(1-b)^{2}$, а намагниченность $m_{n}$ вычислим следующим образом. Пусть $Z_{n}-$ статистическая сумма для отрезка из $\mathrm{n}$ изинговских спинов $\sigma_{1}, \ldots, \sigma_{n}$

$$
\begin{aligned}
Z_{n} & =\sum_{\sigma_{1}, \ldots, \sigma_{n}} \exp \left(K \sum_{i=1}^{n-1} \sigma_{i} \sigma_{i+1}+h_{e x} \sum_{i=1}^{n} \sigma_{i}\right) \\
& =F_{n}(+1)+F_{n}(-1)
\end{aligned}
$$

где

$$
F_{n}\left(\sigma_{n}\right)=\sum_{\sigma_{1}, \ldots, \sigma_{n-1}} \exp \left(K \sum_{i=1}^{n-1} \sigma_{i} \sigma_{i+1}+h_{e x} \sum_{i=1}^{n} \sigma_{i}\right) .
$$

Тогда

$$
m_{n}=\frac{1}{n} \frac{\partial}{\partial h_{e x}} \ln Z_{n}=\frac{1}{n} \frac{F_{n, h}(+1)+F_{n, h}(-1)}{F_{n}(+1)+F_{n}(-1)},
$$

где $F_{n, h}(\sigma)$ - производная $F_{n}(\sigma)$ по $h_{e x}$. Для величин $F_{n}( \pm 1)$ и их производных можно составить рекуррентные соотношения

$$
\begin{aligned}
F_{n+1}(\sigma)= & \sum_{\sigma^{\prime}= \pm 1} F_{n}\left(\sigma^{\prime}\right) e^{K \sigma \sigma^{\prime}+h_{e x} \sigma} \\
= & F_{n}(+1) e^{K \sigma+h_{e x} \sigma}+F_{n}(-1) e^{-K \sigma+h_{e x} \sigma}, \\
F_{n+1, h}(\sigma)= & \left(F_{n, h}(+1)+\sigma F_{n}(+1)\right) e^{K \sigma+h_{e x} \sigma} \\
& +\left(F_{n, h}(-1)+s F_{n}(-1)\right) e^{-K \sigma+h_{e x} \sigma}, \\
F_{1}(+1)= & F_{1, h}(+1)=e^{h_{e x}}, \quad F_{1}(-1)=e^{-h_{e x}}, \\
& F_{1, h}(-1)=-e^{-h_{e x}} .
\end{aligned}
$$

Вводя обозначения

$$
\begin{gathered}
x_{n}=F_{n}(-1) / F_{n}(+1), \quad y_{n}=F_{(n, h)(+1) / F_{n}(+1),} \\
z_{n}=F_{(n, h)(-1) / F_{n}(+1)}
\end{gathered}
$$

получим

$$
\begin{gathered}
m_{n}=\frac{1}{n} \frac{y_{n}+z_{n}}{1+x_{n}}, \\
x_{n+1}=\frac{e^{-2 K}+x_{n}}{1+x_{n} e^{-2 K}} e^{-2 h_{e x}} y_{n+1}=\frac{y_{n}+z_{n} e^{-2 K}}{1+x_{n} e^{-2 K}}+1, \\
z_{n+1}=\frac{z_{n}+y_{n} e^{-2 K}}{1+x_{n} e^{-2 K}} e^{-2 h_{e x}}-x_{n+1}, \\
x_{1}=e^{-2 h_{e x}}, \quad y_{1}=1, \quad z_{1}=-e^{-2 h_{e x}} .
\end{gathered}
$$

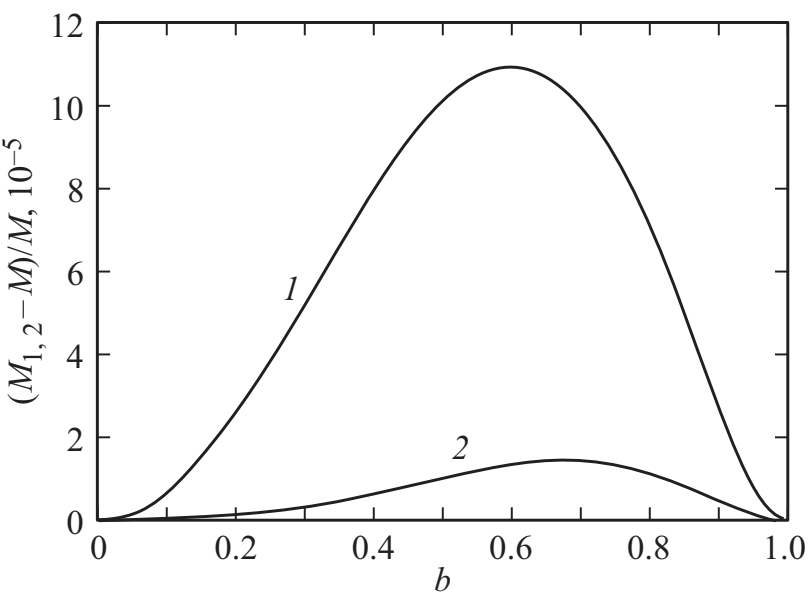

Рис. 1. Относительные разности приближенных и точного решений $\left(M_{1}-M\right) / M$ (кривая 1$)$ и $\left(M_{2}-M\right) / M$ (кривая 2) для одномерной цепочки изинговских спинов с разбавлением по связям. По оси абсцисс - концентрация неразорванных связей $b$.

Сравним теперь решение (16) с приближениями (11) и (13). Взяв в (11) и (13) $q=2$, получим

$$
\begin{gathered}
M_{1}=\operatorname{th}\left(2 x+h_{e x}\right), \\
M_{2}=(1-b)^{2} \operatorname{th}\left(h_{e x}\right)+2 b(1-b) \operatorname{th}\left(y+h_{e x}\right) \\
+b^{2} \operatorname{th}\left(2 y+h_{e x}\right)
\end{gathered}
$$

где $x$ и $y$ находятся из уравнений

$$
\begin{aligned}
\operatorname{th}\left(2 x+h_{e x}\right)= & (1-b) \operatorname{th}\left(x+h_{e x}\right) \\
& +b \frac{\operatorname{sh}\left(2 x+2 h_{e x}\right)}{\operatorname{ch}\left(2 x+2 h_{e x}\right)+e^{-2 K}}
\end{aligned}
$$

и

$$
\begin{aligned}
& (1-b) \operatorname{th}\left(y+h_{e x}\right)+b \operatorname{th}\left(2 y+h_{e x}\right)=(1-b)^{2} \\
& \times \frac{\operatorname{sh}\left(2 h_{e x}\right)}{\operatorname{ch}\left(2 h_{e x}\right)+e^{-2 K}}+2 b(1-b) \frac{\operatorname{sh}\left(y+2 h_{e x}\right)}{\operatorname{ch}\left(y+2 h_{e x}\right)+e^{-2 K} \operatorname{ch}(y)} \\
& +b^{2} \frac{\operatorname{sh}\left(2 y+2 h_{e x}\right)}{\operatorname{ch}\left(2 y+2 h_{e x}\right)+e^{-2 K}} .
\end{aligned}
$$

На рис. 1 показаны относительные разности $\left(M_{1}-M\right) / M$ (кривая 1$)$ и $\left(M_{2}-M\right) / M$ (кривая 2) в зависимости от $b$, вычисленные при $K=0.5$ и $h_{e x}=0.1$. Видно, что биноминальное приближение (13) приводит в данном случае к более точному результату, чем псевдохаотическое приближение (11). Наши расчеты показывают, что и при других значениях параметров $K$ и $h_{e x}$ биноминальное приближение является более точным, чем псевдохаотическое для одномерной разбавленной модели Изинга.

К сожалению, не удается построить точное решение для модели Изинга с разбавлением даже для решетки 


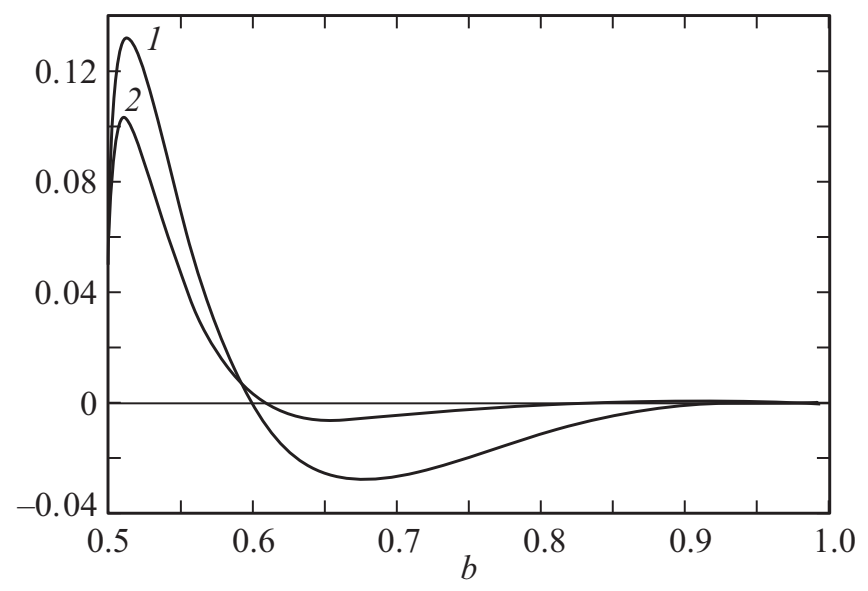

Рис. 2. Разности спонтанных намагниченностей при нулевой температуре и функции $P_{0}(b)$ для псевдохаотического приближения (кривая 1) и биноминального приближения (кривая 2). По оси абсцисс - концентрация неразорванных связей $b$.

Бете с $q>2$. Но для решетки Бете с разбавлением можно сравнительно просто найти вероятность $P_{0}(b)$ того, что случайно взятый узел решетки принадлежит бесконечному кластеру [16]:

$$
P_{0}(b)=1-Z^{q},
$$

где $Z$ 一корень уравнения $\sum_{i=0}^{q-2} Z^{i}=1 / b$.

Известно [16], что средняя спонтанная намагниченность в модели Изинга с разбавлением при $T \rightarrow 0$ $(K \rightarrow \infty)$ должна быть равна $P_{0}(b)$. Поскольку приближения (11) и (13) при $b=1$ есть точные решения для модели Изинга на решетке Бете, то при $b<1$ на них можно смотреть как на приближенные решения для модели Изинга с разбавлением для этой решетки. Поэтому имеет смысл сравнить вероятность $P_{0}(b)$ со спонтанной намагниченностью в приближениях (11) и (13) при $K \rightarrow \infty$.

На рис. 2 показаны разности намагниченности, вычисленной при $T \rightarrow 0$ и вероятности $P_{0}(b)$ для псевдохаотического приближения (10) (кривая 1) и биноминального приближения (13) (кривая 2) для решетки Бете с $q=3$ в зависимости от $b$. Видно, что разность для биноминального приближения меньше, чем для псевдохаотического.

\section{4. Заключение}

Таким образом, в настоящей работе получены следующие результаты. Был сформулирован и обоснован в общей форме метод усреднения по полям взаимодействия, основанный на соотношении (2). Было показано, что этот метод можно применить к разбавленным магнетикам в комбинации с методом самоусреднения [10]. Описанный в работе общий алгоритм был применен для кластеров из одного и двух узлов (формулы (9) и (10)).
С использованием метода усреднения по полям взаимодействия были построены псевдохаотическое (11) и биноминальное (13) приближения для модели Изинга с разбавлением по связям. Точность этих приближений оценивалась с помощью сравнения с точным решением для одномерной модели Изинга с разбавлением по связям. Кроме того, намагниченности при нулевой температуре, полученные в этих приближениях, сравнивались с $P_{0}(b)$ для решетки Бете при $q=3$. Эти сравнения показали, что биноминальное приближение (13) является более точным, чем приближение (11).

\section{Конфликт интересов}

Авторы заявляют, что у них отсутствует конфликт интересов.

\section{Список литературы}

[1] Ю.А. Изюмов, М.В. Медведев. Теория магнитоупорядоченных кристаллов с примесями. Наука, М. (1970). 271 с.

[2] Б.Н. Шалаев. ФТТ 52, 83 (2010).

[3] Е.З. Мейлихов, Р.М. Фарзетдинова. ФТТ 56, 679 (2014).

[4] С. Чандрасекар. Стохастические проблемы в физике и астрономии. ИИЛ, М. (1947). 168 с.

[5] В.И. Белоконь, В.В. Кочегура, Л.Е. Шолпо. Методы палеомагнитных исследований горных пород. Недра, Л. ( 1973). $248 \mathrm{c}$.

[6] В.И. Белоконь, С.В. Семкин. ЖЭТФ 102, 4, 1254 (1992).

[7] С.В. Семкин, В.П. Смагин. ФТТ 55, 892 (2013).

[8] С.В. Семкин, В.П. Смагин. Изв. вузов. Физика, 56, 9 (2013).

[9] Н.B. Callen. Phys. Lett. 4, 161 (1963).

[10] Вик.С. Доценко. Критические явления в спиновых системах с беспорядком. УФН 165, 5, 481 (1995).

[11] С.В. Семкин, В.П. Смагин. ФТТ 56, 1064 (2014).

[12] С.В. Семкин, В.П. Смагин. ФТТ 57, 5, 926 (2015).

[13] С.В. Семкин, В.П. Смагин. ЖЭТФ 148, 729 (2015).

[14] В.П. Смагин, С.В. Семкин. Вестн. ВГУЭС, 4, 122 (2018).

[15] Р. Бэкстер. Точно решаемые модели в статистической механике. Мир, М. (1985). R.J. Baxter. Exactly solved models in statistical mechanics. Academic Press, N.Y. (1982).

[16] Дж. Займан, Модели беспорядка: Теоретическая физика однородно неупорядоченных систем. Мир, М. (1982). $591 \mathrm{c.}$

[17] С.В. Семкин, В.П. Смагин, Е. Г. Гусев. ТМФ 201, 2, 278 (2019).

[18] С.В. Семкин, В.П. Смагин. Изв. вузов. Физика 60, 140 (2017).

Редактор Е.Ю. Флегонтова 